\title{
SYMMETRIC MATRIX PENCILS: CODIMENSION COUNTS AND THE SOLUTION OF A PAIR OF MATRIX EQUATIONS*
}

\author{
ANDRII DMYTRYSHYN ${ }^{\dagger}$, BO KÅGSTRÖM ${ }^{\dagger}$, AND VLADIMIR V. SERGEICHUK ${ }^{\ddagger}$
}

\begin{abstract}
The set of all solutions to the homogeneous system of matrix equations $\left(X^{T} A+\right.$ $\left.A X, X^{T} B+B X\right)=(0,0)$, where $(A, B)$ is a pair of symmetric matrices of the same size, is characterized. In addition, the codimension of the orbit of $(A, B)$ under congruence is calculated. This paper is a natural continuation of the article [A. Dmytryshyn, B. Kågström, and V.V. Sergeichuk. Skew-symmetric matrix pencils: Codimension counts and the solution of a pair of matrix equations. Linear Algebra Appl., 438:3375-3396, 2013.], where the corresponding problems for skew-symmetric matrix pencils are solved. The new results will be useful in the development of the stratification theory for orbits of symmetric matrix pencils.
\end{abstract}

Key words. Pair of symmetric matrices, Matrix equations, Orbits, Codimension.

AMS subject classifications. 15A24, 15A21.

1. Introduction. The goal of this paper is to present the general parameterized solution of the system of matrix equations

$$
\begin{aligned}
& X^{T} A+A X=0, \\
& X^{T} B+B X=0,
\end{aligned}
$$

in which $(A, B)$ is a pair of complex symmetric $\hat{n} \times \hat{n}$ matrice 1 . The set of matrices $X$ that satisfy the system (1.1) forms a vector space whose dimension is also calculated. This dimension plus $\hat{n}$ is equal to the codimension of the orbit of $(A, B)$ with respect to the congruence equivalence relation (see Theorem 2.3).

This article is a logical continuation of the recent paper [14, in which we solved the homogeneous system of matrix equations (1.1) for skew-symmetric matrix pairs and computed the codimensions of skew-symmetric matrix pair orbits under congruence.

\footnotetext{
${ }^{*}$ Received by the editors on May 22, 2013. Accepted for publication on January 6, 2014. Handling Editor: Christian Mehl.

${ }^{\dagger}$ Department of Computing Science and HPC2N, Umeå University, SE-901 87 Umeå, Sweden (andrii@cs.umu.se, bokg@cs.umu.se). Supported by the Swedish Research Council (VR) under grant A0581501, and by eSSENCE, a strategic collaborative e-Science programme funded by the Swedish Government via VR.

${ }^{\ddagger}$ Institute of Mathematics, Tereshchenkivska 3, Kiev, Ukraine (sergeich@imath.kiev.ua). Supported by Fapesp grant 2012/18139-2.

${ }^{1}$ We use $n$ in the definitions of the canonical blocks and $\hat{n} \times \hat{n}$ as the original matrix sizes, where $n \leq \hat{n}$.
} 
Both the papers are directed towards the same audience and to facilitate the reading and the use of results from [14, we keep the notation, the style and the structure of them as similar as possible.

Problems of solving matrix equations as well as computing codimensions of corresponding orbits appear in different branches of science. Recent papers that partially share methods and motivation with this paper include:

- the solution of $X A+A X^{T}=0$ or $X A+A X^{*}=0$ for any square matrix $A$ [4, 7, 6, 19,

- the solution of $A X+X^{T} B=0$ or $A X+X^{*} B=0$ for matrices $A$ and $B$ of compatible sizes 8 ;

- the solution of $A X+B X^{T}=0$ or $A X+B X^{*}=0$ for matrices $A$ and $B$ of compatible sizes [5];

- the solution of a pair of matrix equations $\left(X^{T} A+A X, X^{T} B+B X\right)=(0,0)$ with skew-symmetric $A$ and $B[14$.

The general parameterized solutions of these matrix equations admit to compute the codimensions of corresponding orbits (see also [9, 10, 11, 12]). Besides these cases, the codimensions were computed for contragredient matrix pencils [20] and generalized matrix products 24]. The dimension of the solution space of generalized coupled Sylvester matrix equation $\left(A_{1} X-Y A_{2}, B_{1} X-Y B_{2}\right)=(0,0)$ was derived in [1].

We plan to use the results of [9, 14 to investigate the changes of the canonical forms of skew-symmetric matrix pencils under small perturbations (i.e., the stratification of skew-symmetric matrix pencils).

Symmetric matrix pencils appear often in applications. Examples include the linearization of the quadratic eigenvalue problem coming from the equations of motion or vibration of structural systems [25, 28, symmetric linearizations for symmetric matrix polynomials [21, and the dynamic governing equations of a structure submitted to viscous damping [15]. A Fortran 77 routine for computing a structured staircase form for symmetric matrix pencils is presented in [3]. Miniversal deformations of symmetric matrix pencils are obtained in 10. However, the stratification theory of symmetric matrix pencils is not known yet and is in our field of interests. We recall that the orbit of each matrix pencil has only orbits with higher codimensions in its closure [26, Part III, Theorem 1.7] and thereby the codimensions define the coarse (but not the complete fine) structure of the associated closure hierarchy graph. For more information about deformation and stratification theories, their applications, and software see [16, 17, 23, and references therein.

Using [7, 6, 14] and the results of this paper, Matlab functions for computing codimensions of the congruence and * congruence orbits of matrices, congruence orbits 
of symmetric and skew-symmetric matrix pencils have been developed [13] and added to the Matrix Canonical Structure Toolbox 22 .

The rest of the paper is organized as follows. The main results are presented in Section 2. Without loss of generality, we consider a congruently transformed system (1.1) where the symmetric pair $(A, B)$ is in canonical form under congruence. The general solution of the system (1.1) in explicit form is presented in Theorem 2.1. The dimensions of solution spaces and codimensions of orbits are given in Corollary 2.2 and Theorem 2.3. In Section [3, we prove Theorem 2.1 and Corollary 2.2. Finally, in Section 4 two examples illustrating our results are presented.

All matrices that we consider are over the field of complex numbers.

2. Main result. A matrix pair $(A, B)$ is said to be congruent to $\left(A^{\prime}, B^{\prime}\right)$ if $\left(A^{\prime}, B^{\prime}\right)=S^{T}(A, B) S=\left(S^{T} A S, S^{T} B S\right)$ for some nonsingular $S$ and the set of all matrix pairs congruent to $(A, B)$ is called the orbit of $(A, B)$ under congruence and denoted by $\operatorname{orbit}(A, B)$. Multiplying the equations (1.1) by $S^{T}$ on the left and by $S$ on the right, we obtain

$$
\begin{aligned}
& S^{T} X^{T} S^{-T} \cdot S^{T} A S+S^{T} A S \cdot S^{-1} X S=0, \\
& S^{T} X^{T} S^{-T} \cdot S^{T} B S+S^{T} B S \cdot S^{-1} X S=0,
\end{aligned}
$$

and so the system (1.1) is equivalent to the system

$$
\begin{gathered}
Y^{T} A^{\prime}+A^{\prime} Y=0, \\
Y^{T} B^{\prime}+B^{\prime} Y=0,
\end{gathered}
$$

where $Y:=S^{-1} X S, A^{\prime}:=S^{T} A S$, and $B^{\prime}:=S^{T} B S$. Therefore, it suffices to solve the system (1.1) in which $(A, B)$ is a canonical pair of symmetric matrices under congruence.

For each positive integer $n$, define the $n$-by- $n$ unit matrix $I_{n}$ and the $n$-by- $n$ matrices

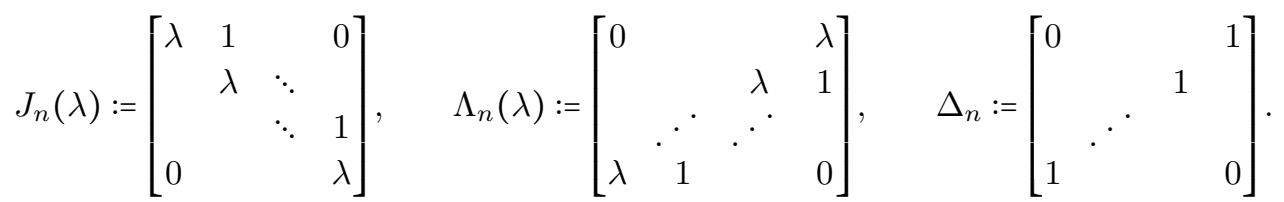

For each nonnegative integer $n$, define the $n$-by- $(n+1)$ matrices

$$
F_{n}:=\left[\begin{array}{cccc}
1 & 0 & & 0 \\
& \ddots & \ddots & \\
0 & & 1 & 0
\end{array}\right], \quad G_{n}:=\left[\begin{array}{cccc}
0 & 1 & & 0 \\
& \ddots & \ddots & \\
0 & & 0 & 1
\end{array}\right] .
$$


Note that $F_{0}$ has size $0 \times 1$, i.e., it has no rows and one (empty) column. Then it is agreed that each $F_{0}$ contributes with a zero column (but no new row) to any matrix it is summed with, e.g., [1] $\oplus F_{0}=\left[\begin{array}{ll}1 & 0\end{array}\right]$ and $[1] \oplus F_{0} \oplus F_{0}=\left[\begin{array}{lll}1 & 0 & 0\end{array}\right]$. Note that $G_{0}=F_{0}$.

Define the direct sum of matrix pairs as follows:

$$
(A, B) \oplus(C, D)=(A \oplus C, B \oplus D) .
$$

The canonical form of a pair of symmetric complex matrices is given in [27]: such a pair is congruent to a direct sum, determined uniquely up to permutation of summands, of pairs of the form

$$
\begin{aligned}
H_{n}(\lambda) & :=\left(\Delta_{n}, \Lambda_{n}(\lambda)\right), \quad \lambda \in \mathbb{C}, \\
K_{n} & :=\left(\Lambda_{n}(0), \Delta_{n}\right), \\
L_{n} & :=\left(\left[\begin{array}{cc}
0 & F_{n}^{T} \\
F_{n} & 0
\end{array}\right],\left[\begin{array}{cc}
0 & G_{n}^{T} \\
G_{n} & 0
\end{array}\right]\right) .
\end{aligned}
$$

Thus, each pair of symmetric matrices is congruent to a direct sum

$$
(A, B)=\bigoplus_{i=1}^{a} H_{h_{i}}\left(\lambda_{i}\right) \oplus \bigoplus_{j=1}^{b} K_{k_{j}} \oplus \bigoplus_{r=1}^{c} L_{l_{r}},
$$

consisting of direct summands of three types.

In the following, we define several parameter matrices, whose nonzero entries $p_{1}, p_{2}, p_{3}, \ldots$ are independent parameters; they will be used to express the set of solutions of (1.1), where $(A, B)$ is in canonical form (2.4).

- The $m \times n$ Hankel matrices

$$
\begin{aligned}
& P_{m n}:=\left[\begin{array}{cccccc}
p_{1} & p_{2} & p_{3} & p_{4} & & \\
p_{2} & p_{3} & p_{4} & & . & \\
p_{3} & p_{4} & & . & & \\
p_{4} & & . & & & \\
& . & & & & p_{m+n-2} \\
& & & & p_{m+n-2} & p_{m+n-1}
\end{array}\right] \text { (a dense Hankel matrix), }
\end{aligned}
$$

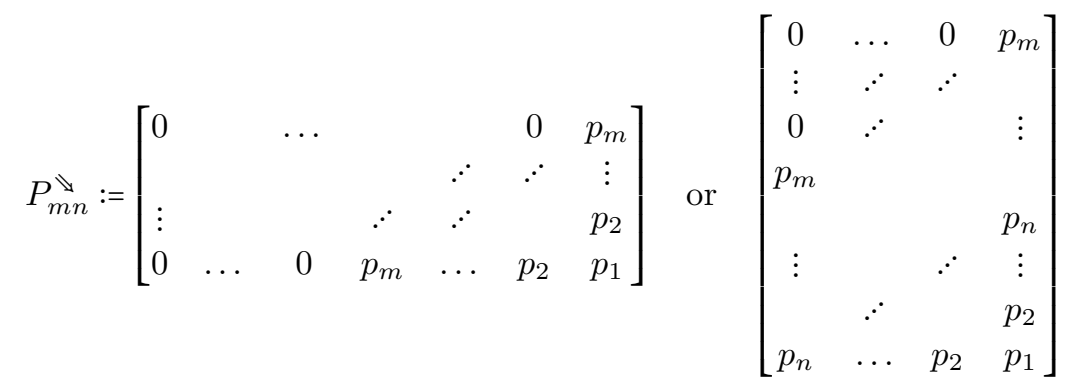


if $m \leqslant n$ or $m>n$, respectively.

- The $m \times n$ Toeplitz matrices

$$
P_{m n}^{\pi}:=\left[\begin{array}{ccccccc}
0 & \ldots & 0 & p_{m} & \cdots & p_{2} & p_{1} \\
& & & \ddots & \ddots & & p_{2} \\
\vdots & & & & \ddots & \ddots & \vdots \\
0 & & \ldots & & & 0 & p_{m}
\end{array}\right] \text { or }\left[\begin{array}{cccc}
p_{n} & \cdots & p_{2} & p_{1} \\
0 & \ddots & & p_{2} \\
& \ddots & \ddots & \vdots \\
& & \ddots & p_{n} \\
\vdots & & & 0 \\
& & & \vdots \\
0 & \ldots & & 0
\end{array}\right]
$$

if $m \leqslant n$ or $m>n$, respectively.

- The $m \times n$ banded Toeplitz matrices

$$
\begin{aligned}
& P_{m n}^{\leftrightarrow}:=\left[\begin{array}{ccccc}
p_{1} & \ldots & p_{n-m+1} & & 0 \\
& \ddots & & \ddots & \\
0 & & p_{1} & \ldots & p_{n-m+1}
\end{array}\right] \text { if } m \leqslant n \text { and } 0 \text { if } m>n, \\
& P_{m n}^{\uparrow}:=0 \text { if } m<n \text { and }\left[\begin{array}{ccc}
p_{1} & & 0 \\
\vdots & \ddots & \\
& & p_{1} \\
p_{m-n+1} & & \\
& \ddots & \vdots \\
0 & & p_{m-n+1}
\end{array}\right] \text { if } m \geqslant n \text {. }
\end{aligned}
$$

- For each $\lambda \in \mathbb{C}$, define the $m \times n$ matrix with parameters $p_{1}, \ldots, p_{n}$ :

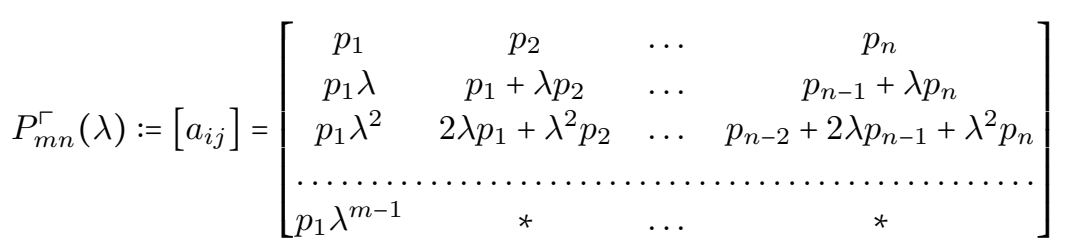

whose rows are determined recurrently via $a_{i j}=\lambda a_{i-1, j}+a_{i-1, j-1}$.

- For all matrices $P_{m n}, P_{m n}^{\Downarrow}, P_{m n}^{\nearrow}, P_{m n}^{\leftrightarrow}, P_{m n}^{\uparrow}, P_{m n}^{\ulcorner}$defined above, we denote by

$$
Q_{m n}, Q_{m n}^{\Downarrow}, Q_{m n}^{\nearrow}, Q_{m n}^{\leftrightarrow}, Q_{m n}^{\uparrow}, Q_{m n}^{\ulcorner} \quad \text { and } \quad R_{m n}, R_{m n}^{\Downarrow}, R_{m n}^{\nearrow}, R_{m n}^{\leftrightarrow}, R_{m n}^{\uparrow}, R_{m n}^{\ulcorner}
$$

the parameter matrices that are obtained by replacing all parameters $p_{i}$ with $q_{i}$ and $r_{i}$, respectively.

We say that a parameter matrix $Q\left(\delta_{1}, \ldots, \delta_{s}\right)$ is obtained by reparametrization of a parameter matrix $\mathcal{P}\left(\varepsilon_{1}, \ldots, \varepsilon_{s}\right)$ and write $\mathcal{P}\left(\varepsilon_{1}, \ldots, \varepsilon_{s}\right) \simeq \mathcal{Q}\left(\delta_{1}, \ldots, \delta_{s}\right)$ if there exists a permutation $\sigma$ of $\{1, \ldots, s\}$ such that $\mathcal{P}\left(\delta_{\sigma(1)}, \ldots, \delta_{\sigma(s)}\right)=\mathcal{Q}\left(\delta_{1}, \ldots, \delta_{s}\right)$. In 
other words, $\mathcal{P}\left(\varepsilon_{1}, \ldots, \varepsilon_{s}\right)$ and $Q\left(\delta_{1}, \ldots, \delta_{s}\right)$ coincide up to relettering of parameters $\left(\varepsilon_{i}=\delta_{\sigma(i)}\right)$.

In the following, let $(A, B)$ be a canonical matrix pair and let

$$
(A, B)=\left(A_{1}, B_{1}\right) \oplus \cdots \oplus\left(A_{t}, B_{t}\right), \quad t:=a+b+c,
$$

be its decomposition (2.4). In Theorem 2.1, we prove that the set of all solutions of the system (1.1) consists of all matrices $\mathcal{P}\left(a_{1}, \ldots, a_{s}\right)$ with $\left(a_{1}, \ldots a_{s}\right) \in \mathbb{C}^{s}$ in which $\mathcal{P}\left(\pi_{1}, \ldots, \pi_{s}\right)$ is a parameter matrix that has the same size as $A$ and $B$ and the same partition into blocks:

$$
\mathcal{P}=\left[\begin{array}{ccc}
\mathcal{P}_{11} & \ldots & \mathcal{P}_{1 t} \\
\vdots & \ddots & \vdots \\
\mathcal{P}_{t 1} & \ldots & \mathcal{P}_{t t}
\end{array}\right], \quad \text { size } \mathcal{P}_{i i}=\operatorname{size} A_{i}=\operatorname{size} B_{i}
$$

The blocks of $\mathcal{P}$ satisfy the condition:

$$
\text { the sets of parameters of } \mathcal{P}_{i j} \text { and } \mathcal{P}_{i^{\prime} j^{\prime}} \text { are disjoint if }(i, j) \neq\left(j^{\prime}, i^{\prime}\right)
$$

and are determined by $(A, B)$ as follows. Write

$$
\mathcal{P}\left(\left(A_{i}, B_{i}\right)\right):=\mathcal{P}_{i i}, \quad \mathcal{P}\left(\left(A_{i}, B_{i}\right),\left(A_{j}, B_{j}\right)\right):=\left(\mathcal{P}_{j i}, \mathcal{P}_{i j}\right) \quad \text { if } i<j,
$$

then:

(i) The diagonal blocks of $\mathcal{P}$ are defined up to reparametrization by the following conditions:

$$
\begin{aligned}
\mathcal{P}\left(H_{n}(\lambda)\right) & =0, \\
\mathcal{P}\left(K_{n}\right) & =0 \\
\mathcal{P}\left(L_{n}\right) & \simeq\left[\begin{array}{ll}
\alpha I_{n+1} & 0_{n+1, n} \\
0_{n, n+1} & -\alpha I_{n}
\end{array}\right] .
\end{aligned}
$$

(ii) The off-diagonal blocks of $\mathcal{P}$ whose horizontal and vertical strips contain summands of $(A, B)$ of the same type are defined up to reparametrization by

$$
\begin{aligned}
& \mathcal{P}\left(H_{n}(\lambda), H_{m}(\mu)\right) \simeq \begin{cases}(0,0) & \text { if } \lambda \neq \mu, \\
\left(-P_{m n}^{\lambda}, P_{n m}^{\lambda}\right) & \text { if } \lambda=\mu,\end{cases} \\
& \mathcal{P}\left(K_{n}, K_{m}\right) \simeq\left(-P_{m n}^{\nearrow}, P_{n m}^{\lambda}\right),
\end{aligned}
$$

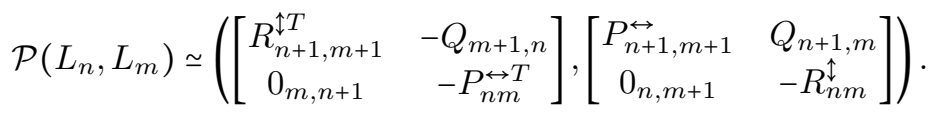


(iii) The off-diagonal blocks of $\mathcal{P}$ whose horizontal and vertical strips contain summands of $(A, B)$ of different types are defined up to reparametrization by

$$
\begin{aligned}
& \mathcal{P}\left(H_{n}(\lambda), K_{m}\right)=(0,0), \\
& \mathcal{P}\left(H_{n}(\lambda), L_{m}\right) \simeq\left(\left[\begin{array}{c}
-P_{m+1, n}^{\ulcorner}(\lambda) \\
0_{m n}
\end{array}\right],\left[\begin{array}{ll}
0_{n, m+1} & \Delta_{n} P_{m+1, n}^{\ulcorner T}(\lambda) F_{m}^{T}
\end{array}\right]\right), \\
& \mathcal{P}\left(K_{n}, L_{m}\right) \simeq\left(\left[\begin{array}{c}
-P_{n, m+1}^{\Downarrow T} \\
0_{m n}
\end{array}\right],\left[\begin{array}{ll}
0_{n, m+1} & \Delta_{n} P_{n, m+1}^{\Downarrow} G_{m}^{T}
\end{array}\right]\right) .
\end{aligned}
$$

Note that if any of the matrix pairs (2.12)-2.17) have blocks denoted by the same letter, then these blocks have the same set of independent parameters, e.g., in (2.14) the blocks $P_{n m}^{\leftrightarrow T}$ and $P_{n+1, m+1}^{\leftrightarrow}$ both have $m-n+1$ independent parameters $p_{1}, \ldots, p_{m-n+1}$, the blocks $Q_{m+1, n}$ and $Q_{n+1, m}$ both have $m+n$ independent parameters $q_{1}, \ldots, q_{m+n}$, and the blocks $R_{n+1, m+1}^{\uparrow T}$ and $R_{n m}^{\downarrow}$ both have $n-m+1$ independent parameters $r_{1}, \ldots, r_{n-m+1}$.

THEOREM 2.1. Let the system (1.1) be given by the canonical pair (2.4) of symmetric matrices for congruence. Let $\mathcal{P}\left(\pi_{1}, \ldots, \pi_{s}\right)$ be a parameter matrix (2.6) whose blocks are defined in (2.7)-2.17). Then

$$
\left\{\mathcal{P}\left(a_{1}, \ldots, a_{s}\right) \mid\left(a_{1}, \ldots, a_{s}\right) \in \mathbb{C}^{s}\right\}
$$

is the set of all solutions of the system (1.1).

COROllary 2.2. If the system (1.1) is given by the canonical pair (2.4), then the dimension of its solution space (2.18) is equal to the sum

$$
d_{(A, B)}=d_{H}+d_{K}+d_{L}+d_{H H}+d_{K K}+d_{L L}+d_{H K}+d_{H L}+d_{K L}
$$

whose summands correspond to

- the direct summands of (2.4) of the same type:

$$
d_{H}:=0, \quad d_{K}:=0, \quad d_{L}:=c
$$

- the pairs of direct summands of (2.4) of the same type:

$$
\begin{gathered}
d_{H H}:=\sum_{\substack{i \leqslant i^{\prime} \\
\lambda_{i}=\lambda_{i^{\prime}}}} \min \left(h_{i}, h_{i^{\prime}}\right), \quad d_{K K}:=\sum_{j \leqslant j^{\prime}} \min \left(k_{j}, k_{j^{\prime}}\right), \\
d_{L L}:=\sum_{r \leqslant r^{\prime}}\left(2 \max \left(l_{r}, l_{r^{\prime}}\right)+\varepsilon_{r r^{\prime}}\right), \quad \text { in which } \varepsilon_{r r^{\prime}}:= \begin{cases}2 & \text { if } l_{r}=l_{r^{\prime}}, \\
1 & \text { if } l_{r} \neq l_{r^{\prime}}\end{cases}
\end{gathered}
$$


- the pairs of direct summands of (2.4) of different types:

$$
d_{H K}:=0, \quad d_{H L}:=c \sum_{i} h_{i}, \quad d_{K L}:=c \sum_{j} k_{j} .
$$

The set of matrix pairs that are congruent to a pair $(A, B)$ of $\hat{n} \times \hat{n}$ symmetric matrices is a manifold in the complex $\hat{n}(\hat{n}+1)$ dimensional space of all pairs of $\hat{n} \times \hat{n}$ symmetric matrices. This manifold is the orbit of $(A, B)$ under the action of congruence. The vector space

$$
T(A, B):=\left\{U^{T}(A, B)+(A, B) U \mid U \in \mathbb{C}^{\hat{n} \times \hat{n}}\right\}
$$

is the tangent space to the congruence orbit of $(A, B)$ at the point $(A, B)$ since

$$
(I+\varepsilon U)^{T}(A, B)(I+\varepsilon U)=(A, B)+\varepsilon\left(U^{T}(A, B)+(A, B) U\right)+\varepsilon^{2} U^{T}(A, B) U
$$

for all $\hat{n}$-by- $\hat{n}$ matrices $U$ and each $\varepsilon \in \mathbb{C}$. The dimension of the orbit of $(A, B)$ is the dimension of its tangent space at the point $(A, B)$; it is well defined because the dimensions of tangent spaces at all points of the orbit are equal (see [2, Chapter IV, Corollary $1.5])$. The codimension of the orbit of $(A, B)$ (denoted by $\operatorname{codim}(\operatorname{orbit}(A, B)))$ is the dimension of the normal space to its orbit at the point $(A, B)$, which is equal to $\hat{n}(\hat{n}+1)$ (the dimension of the space of all pairs of $\hat{n} \times \hat{n}$ symmetric matrices) minus the dimension of the orbit of $(A, B)$. Note that the orthogonality in the space of all pairs of $\hat{n} \times \hat{n}$ symmetric matrices is defined with respect to the Frobenius inner product

$$
\langle(A, B),(C, D)\rangle=\operatorname{tr}\left(A C^{*}+B D^{*}\right),
$$

where $\operatorname{tr}(X)$ denotes the trace of a square matrix $X$.

The formula (2.19) admits to calculate the codimension of congruence orbit of the canonical pair (2.4) due to the following theorem.

THEOREM 2.3. The codimension of the congruence orbit of a pair $(A, B)$ of $\hat{n} \times \hat{n}$ symmetric matrices is equal to

$$
d_{(A, B)}+\hat{n},
$$

in which $d_{(A, B)}$ is the dimension of the solution space of system (1.1).

Proof. Define the mapping

$$
f: \mathbb{C}^{\hat{n} \times \hat{n}} \rightarrow T(A, B), \quad X \mapsto X^{T}(A, B)+(A, B) X,
$$

where $T(A, B)$ is the tangent space at the point $(A, B) \in \mathbb{C}_{s}^{\hat{n} \times \hat{n}} \times \mathbb{C}_{s}^{\hat{n} \times \hat{n}}$ (see (2.20)), and $\mathbb{C}_{s}^{\hat{n} \times \hat{n}}$ is the space of symmetric $\hat{n} \times \hat{n}$ matrices. 
Since this mapping is a surjective homomorphism, $\operatorname{dim} \mathbb{C}^{\hat{n} \times \hat{n}}=\operatorname{dim} T(A, B)+$ $\operatorname{dim} V(A, B)$, where $V(A, B):=\left\{X \in \mathbb{C}^{\hat{n} \times \hat{n}} \mid X^{T}(A, B)+(A, B) X=0\right\}$. We recall that $\operatorname{dim} V(A, B)=d_{(A, B)}$, e.g., computed as in (2.19). At every point $(A, B)$ we have the decomposition

$$
\mathbb{C}_{s}^{\hat{n} \times \hat{n}} \times \mathbb{C}_{s}^{\hat{n} \times \hat{n}}=T(A, B) \oplus N(A, B),
$$

in which $N(A, B)$ is the normal space at the point $(A, B)$ with respect to the inner product defined in (2.21). Therefore,

$$
\begin{aligned}
\operatorname{codim}(\operatorname{orbit}(A, B)) & =\operatorname{dim} N(A, B)=\operatorname{dim}\left(\mathbb{C}_{s}^{\hat{n} \times \hat{n}} \times \mathbb{C}_{s}^{\hat{n} \times \hat{n}}\right)-\operatorname{dim} T(A, B) \\
& =\operatorname{dim}\left(\mathbb{C}_{s}^{\hat{n} \times \hat{n}} \times \mathbb{C}_{s}^{\hat{n} \times \hat{n}}\right)-\operatorname{dim} \mathbb{C}^{\hat{n} \times \hat{n}}+\operatorname{dim} V(A, B) \\
& =\hat{n}^{2}+\hat{n}-\hat{n}^{2}+d_{(A, B)}=d_{(A, B)}+\hat{n} .
\end{aligned}
$$

3. Proof of Theorem 2.1 and Corollary 2.2, Let $(M, N)$ and $\left(M^{\prime}, N^{\prime}\right)$ be two pairs of symmetric matrices such that the sizes of $M$ and $N$ coincide and the sizes of $M^{\prime}$ and $N^{\prime}$ coincide. Define two systems of matrix equations:

$$
\begin{array}{rll}
\operatorname{syst}((M, N)): & X^{T} M+M X=0, & X^{T} N+N X=0 ; \\
\operatorname{syst}\left((M, N),\left(M^{\prime}, N^{\prime}\right)\right): & Z M^{\prime}+M Y=0, & Z N^{\prime}+N Y=0 .
\end{array}
$$

Lemma 3.1. Let

$$
(A, B)=\left(A_{1}, B_{1}\right) \oplus \cdots \oplus\left(A_{t}, B_{t}\right)
$$

be a pair of symmetric matrices and let $X=\left[X_{i j}\right]_{i, j=1}^{t}$ be a complex block matrix such that the sizes of $A_{i}, B_{i}$, and $X_{i i}$ are equal for every $i=1, \ldots, t$. Then $X$ is a solution of (1.1) (i.e., $\operatorname{syst}((A, B)))$ if and only if each diagonal block $X_{i i}$ is a solution of $\operatorname{syst}\left(\left(A_{i}, B_{i}\right)\right)$ and each off-diagonal block pair $\left(X_{j i}, X_{i j}\right)=\left(Z_{j i}^{T}, Y_{i j}\right)$ with $i \leqslant j$, where $\left(Z_{j i}, Y_{i j}\right)$ is a solution of $\operatorname{syst}\left(\left(A_{i}, B_{i}\right),\left(A_{j}, B_{j}\right)\right)$.

Proof. It is enough to consider $t=2$. Partitioning the unknown matrix $X$ we rewrite system (1.1) as follows

$$
\begin{aligned}
& {\left[\begin{array}{ll}
X_{11}^{T} & X_{21}^{T} \\
X_{12}^{T} & X_{22}^{T}
\end{array}\right]\left[\begin{array}{cc}
A_{1} & 0 \\
0 & A_{2}
\end{array}\right]+\left[\begin{array}{cc}
A_{1} & 0 \\
0 & A_{2}
\end{array}\right]\left[\begin{array}{ll}
X_{11} & X_{12} \\
X_{21} & X_{22}
\end{array}\right]=\left[\begin{array}{ll}
0 & 0 \\
0 & 0
\end{array}\right],} \\
& {\left[\begin{array}{ll}
X_{11}^{T} & X_{21}^{T} \\
X_{12}^{T} & X_{22}^{T}
\end{array}\right]\left[\begin{array}{cc}
B_{1} & 0 \\
0 & B_{2}
\end{array}\right]+\left[\begin{array}{cc}
B_{1} & 0 \\
0 & B_{2}
\end{array}\right]\left[\begin{array}{ll}
X_{11} & X_{12} \\
X_{21} & X_{22}
\end{array}\right]=\left[\begin{array}{ll}
0 & 0 \\
0 & 0
\end{array}\right] .}
\end{aligned}
$$

Multiplying the matrices we obtain

$$
\begin{aligned}
& {\left[\begin{array}{ll}
X_{11}^{T} A_{1}+A_{1} X_{11} & X_{21}^{T} A_{2}+A_{1} X_{12} \\
X_{12}^{T} A_{1}+A_{2} X_{21} & X_{22}^{T} A_{2}+A_{2} X_{22}
\end{array}\right]=\left[\begin{array}{ll}
0 & 0 \\
0 & 0
\end{array}\right],} \\
& {\left[\begin{array}{ll}
X_{11}^{T} B_{1}+B_{1} X_{11} & X_{21}^{T} B_{2}+B_{1} X_{12} \\
X_{12}^{T} B_{1}+B_{2} X_{21} & X_{22}^{T} B_{2}+B_{2} X_{22}
\end{array}\right]=\left[\begin{array}{ll}
0 & 0 \\
0 & 0
\end{array}\right],}
\end{aligned}
$$


where the systems on the diagonals are $\operatorname{syst}\left(\left(A_{1}, B_{1}\right)\right)$ and $\operatorname{syst}\left(\left(A_{2}, B_{2}\right)\right)$, and the system in the position $(1,2)$ is $\operatorname{syst}\left(\left(A_{1}, B_{1}\right),\left(A_{2}, B_{2}\right)\right)$ with $\left(X_{21}^{T}, X_{12}\right)=(Z, Y) \square$

If $(A, B)$ is the pair (2.5) in canonical form, then it is enough to solve

$$
\operatorname{syst}\left(\left(A_{i}, B_{i}\right)\right), \quad \operatorname{syst}\left(\left(A_{i}, B_{i}\right),\left(A_{j}, B_{j}\right)\right) \text { with } i<j,
$$

in which all $\left(A_{i}, B_{i}\right)$ are of the form (2.1)-(2.3). All possible cases are considered in Sections 3.1 to 3.7 .

3.1. Solution of $\operatorname{syst}\left(H_{n}(\lambda)\right)$ and $\operatorname{syst}\left(K_{n}\right)$. The system syst $\left(H_{n}(\lambda)\right)$ has the form

$$
X^{T} \Delta_{n}+\Delta_{n} X=0, \quad X^{T} \Lambda_{n}(\lambda)+\Lambda_{n}(\lambda) X=0 .
$$

From the first equation we have $X^{T}=-\Delta_{n} X \Delta_{n}$. Substituting it in the second equation, multiplying the equation by $\Delta_{n}$ from the left side, and using that $\Delta_{n} \Delta_{n}=I_{n}$ and $\Delta_{n} \Lambda_{n}(\lambda)=J_{n}(\lambda)$ we obtain

$$
X^{T}+\Delta_{n} X \Delta_{n}=0, \quad-X J_{n}(\lambda)+J_{n}(\lambda) X=0 .
$$

Since

$$
-X J_{n}(\lambda)+J_{n}(\lambda) X=-\lambda X-X J_{n}(0)+J_{n}(0) X+\lambda X=-X J_{n}(0)+J_{n}(0) X,
$$

the solution does not depend on $\lambda$. By [18, Chapter VIII], the equation $X J_{n}(0)=$ $J_{n}(0) X$ has the solution $X=P_{n n}^{\lambda}$ and so $X^{T}=\Delta_{n} X \Delta_{n}=-X^{T}$. Thus, the only solution of (3.1) is $X=0$, which proves (2.9) due to Lemma 3.1. Since (3.1) holds, $\operatorname{syst}\left(K_{n}\right)$ has the same solution as the system $\operatorname{syst}\left(H_{n}(0)\right)$, so (2.10) is proven too. Thus, $d_{H}=d_{K}=0$ and by Theorem $2.3 \operatorname{codim}\left(\operatorname{orbit} H_{n}(\lambda)\right)=\operatorname{codim}\left(\operatorname{orbit} K_{n}\right)=n$.

3.2. Solution of $\operatorname{syst}\left(L_{n}\right)$. The system $\operatorname{syst}\left(L_{n}\right)$ has the form

$$
\begin{aligned}
& {\left[\begin{array}{cc}
X_{11}^{T} & X_{21}^{T} \\
X_{12}^{T} & X_{22}^{T}
\end{array}\right]\left[\begin{array}{cc}
0 & F_{n}^{T} \\
F_{n} & 0
\end{array}\right]+\left[\begin{array}{cc}
0 & F_{n}^{T} \\
F_{n} & 0
\end{array}\right]\left[\begin{array}{ll}
X_{11} & X_{12} \\
X_{21} & X_{22}
\end{array}\right]=\left[\begin{array}{ll}
0 & 0 \\
0 & 0
\end{array}\right],} \\
& {\left[\begin{array}{cc}
X_{11}^{T} & X_{21}^{T} \\
X_{12}^{T} & X_{22}^{T}
\end{array}\right]\left[\begin{array}{cc}
0 & G_{n}^{T} \\
G_{n} & 0
\end{array}\right]+\left[\begin{array}{cc}
0 & G_{n}^{T} \\
G_{n} & 0
\end{array}\right]\left[\begin{array}{ll}
X_{11} & X_{12} \\
X_{21} & X_{22}
\end{array}\right]=\left[\begin{array}{ll}
0 & 0 \\
0 & 0
\end{array}\right],}
\end{aligned}
$$

in which $X$ is partitioned conformally with the $2 \times 2$ block structure of $L_{n}$. Multiplying the matrices we have

$$
\begin{aligned}
& {\left[\begin{array}{cc}
X_{21}^{T} F_{n}+F_{n}^{T} X_{21} & X_{11}^{T} F_{n}^{T}+F_{n}^{T} X_{22} \\
X_{22}^{T} F_{n}+F_{n} X_{11} & X_{12}^{T} F_{n}^{T}+F_{n} X_{12}
\end{array}\right]=\left[\begin{array}{ll}
0 & 0 \\
0 & 0
\end{array}\right],} \\
& {\left[\begin{array}{cc}
X_{21}^{T} G_{n}+G_{n}^{T} X_{21} & X_{11}^{T} G_{n}^{T}+G_{n}^{T} X_{22} \\
X_{22}^{T} G_{n}+G_{n} X_{11} & X_{12}^{T} G_{n}^{T}+G_{n} X_{12}
\end{array}\right]=\left[\begin{array}{ll}
0 & 0 \\
0 & 0
\end{array}\right] .}
\end{aligned}
$$


Since the pairs of blocks at positions $(1,2)$ and $(2,1)$ are equal up to the transposition, (3.2) decomposes into three independent subsystems.

First consider the subsystem corresponding to the $(1,1)$-blocks:

$$
\begin{aligned}
& X_{21}^{T} F_{n}+F_{n}^{T} X_{21}=0, \\
& X_{21}^{T} G_{n}+G_{n}^{T} X_{21}=0 .
\end{aligned}
$$

To satisfy the first equation of (3.3), $X_{21}$ must have the form

$$
X_{21}=\left[\begin{array}{cccccc}
0 & x_{12} & x_{13} & \ldots & x_{1 n} & 0 \\
-x_{12} & 0 & x_{23} & \ldots & x_{2 n} & 0 \\
-x_{13} & -x_{23} & 0 & \ldots & x_{3 n} & 0 \\
\vdots & \vdots & \vdots & \ddots & \vdots & \vdots \\
-x_{1 n} & -x_{2 n} & -x_{3 n} & \ldots & 0 & 0
\end{array}\right] .
$$

The matrix $X_{21}$ without the last column is skew symmetric. Substituting $X_{21}$ in the second equation of (3.3), we obtain

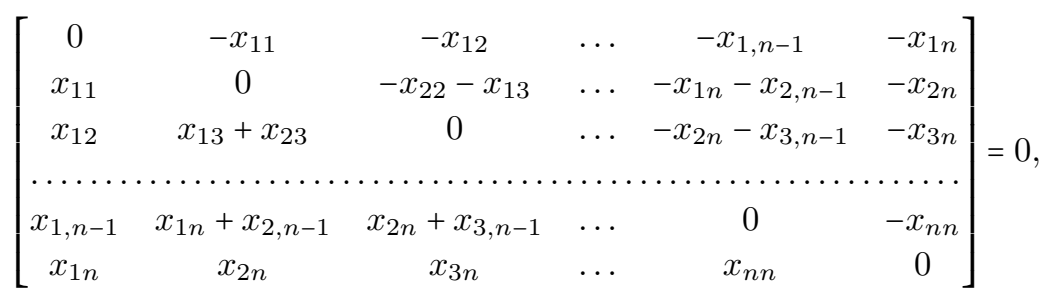

which implies that $X_{21}=0$.

Now consider the subsystem corresponding to the $(2,2)$-blocks:

$$
\begin{aligned}
& X_{12}^{T} F_{n}^{T}+F_{n} X_{12}=0, \\
& X_{12}^{T} G_{n}^{T}+G_{n} X_{12}=0 .
\end{aligned}
$$

To satisfy the first equation of (3.4), $X_{12}^{T}$ must have the form

$$
X_{12}^{T}=\left[\begin{array}{cccccc}
0 & x_{12} & x_{13} & \ldots & x_{1 n} & x_{1, n+1} \\
-x_{12} & 0 & x_{23} & \ldots & x_{2 n} & x_{2, n+1} \\
-x_{13} & -x_{23} & 0 & \ldots & x_{3 n} & x_{3, n+1} \\
\vdots & \vdots & \vdots & \ddots & \vdots & \vdots \\
-x_{1 n} & -x_{2 n} & -x_{3 n} & \ldots & 0 & x_{n, n+1}
\end{array}\right] .
$$

Substituting it in the second equation of (3.4), we obtain

$$
\left[\begin{array}{ccccc}
2 x_{12} & x_{13} & \ldots & & x_{1, n+1}-x_{2 n} \\
x_{13} & 2 x_{23} & \ddots & & x_{2, n+1}-x_{3 n} \\
\vdots & \ddots & \ddots & \ddots & \vdots \\
x_{1 n}-x_{2, n-1} & & \ddots & 2 x_{n-1, n} & x_{n-1, n+1} \\
x_{1, n+1}-x_{2 n} & & \ldots & x_{n-1, n+1} & 2 x_{n, n+1}
\end{array}\right]=0,
$$


and so $X_{21}^{T}=0$.

By [14, Section 3.2] the solution of the off-diagonal subsystem

$$
\begin{aligned}
X_{22}^{T} F_{n}+F_{n} X_{11} & =0, \\
X_{22}^{T} G_{n}+G_{n} X_{11} & =0,
\end{aligned}
$$

is $X_{11}=\alpha I_{n+1}$ and $X_{22}=-\alpha I_{n}$, where $\alpha$ is a parameter.

Summing up, the solution of system (3.2) is

$$
X=\left[\begin{array}{cc}
\alpha I_{n+1} & 0_{n+1, n} \\
0_{n, n+1} & -\alpha I_{n}
\end{array}\right],
$$

which together with Lemma 3.1 ensure (2.14). We have just one independent parameter in $X$, thus $d_{L}=1$, and by Theorem 2.3 , codim(orbit $\left.L_{n}\right)=2 n+1+1=2 n+2$.

3.3. Solution of $\operatorname{syst}\left(H_{n}(\lambda), H_{m}(\mu)\right)$ and $\operatorname{syst}\left(K_{n}, K_{m}\right)$. In this section, we calculate off-diagonal blocks of the solution of the system (1.1) that correspond to the diagonal blocks $H_{n}(\lambda)$ and $H_{m}(\mu)$. The system syst $\left(H_{n}(\lambda), H_{m}(\mu)\right)$ has the following form

$$
Z \Delta_{m}+\Delta_{n} Y=0, \quad Z \Lambda_{m}(\mu)+\Lambda_{n}(\lambda) Y=0,
$$

where $Z$ and $Y$ are the unknown $n$-by- $m$ matrices.

From the first equation we have $Z=-\Delta_{n} Y \Delta_{m}$. We substitute this value of $Z$ in the second equation, multiplay the equation by $\Delta_{n}$, and using that $\Delta_{n} \Delta_{n}=I_{n}$ and $\Delta_{n} \Lambda_{n}(\lambda)=J_{n}(\lambda)$ we obtain:

$$
Z+\Delta_{n} Y \Delta_{m}=0, \quad-Y J_{m}(\mu)+J_{n}(\lambda) Y=0 .
$$

By [18, Chapter VIII] the equation $Y J_{m}(\mu)=J_{n}(\lambda) Y$ has the solution $Y=0$ if $\lambda \neq \mu$ and $Y=P_{n m}^{\nearrow}$ if $\lambda=\mu$. Summing up, we have

$$
Z^{T}=\left\{\begin{array}{ll}
0 & \text { if } \lambda \neq \mu, \\
-P_{m n}^{X} & \text { if } \lambda=\mu,
\end{array} \text { and } \quad Y= \begin{cases}0 & \text { if } \lambda \neq \mu, \\
P_{n m}^{X} & \text { if } \lambda=\mu,\end{cases}\right.
$$

which together with Lemma 3.1 prove (2.12). Note that the system $\operatorname{syst}\left(K_{n}, K_{m}\right)$ has the same solution as $\operatorname{syst}\left(H_{n}(0), H_{m}(0)\right)$, and thus, (2.13) is justified too. The numbers of independent parameters in the solutions are equal to $d_{K K}=\min (n, m)$ and

$$
d_{H H}= \begin{cases}0 & \text { if } \lambda \neq \mu, \\ \min (n, m) & \text { if } \lambda=\mu .\end{cases}
$$


3.4. Solution of $\operatorname{syst}\left(L_{n}, L_{m}\right)$. In this section, we calculate off-diagonal blocks of the solution of the system (1.1) that correspond to the diagonal blocks $L_{n}$ and $L_{m}$. The system syst $\left(L_{n}, L_{m}\right)$ has the form

$$
\begin{aligned}
& {\left[\begin{array}{ll}
Z_{11} & Z_{12} \\
Z_{21} & Z_{22}
\end{array}\right]\left[\begin{array}{cc}
0 & F_{m}^{T} \\
F_{m} & 0
\end{array}\right]+\left[\begin{array}{cc}
0 & F_{n}^{T} \\
F_{n} & 0
\end{array}\right]\left[\begin{array}{ll}
Y_{11} & Y_{12} \\
Y_{21} & Y_{22}
\end{array}\right]=\left[\begin{array}{ll}
0 & 0 \\
0 & 0
\end{array}\right],} \\
& {\left[\begin{array}{cc}
Z_{11} & Z_{12} \\
Z_{21} & Z_{22}
\end{array}\right]\left[\begin{array}{cc}
0 & G_{m}^{T} \\
G_{m} & 0
\end{array}\right]+\left[\begin{array}{cc}
0 & G_{n}^{T} \\
G_{n} & 0
\end{array}\right]\left[\begin{array}{ll}
Y_{11} & Y_{12} \\
Y_{21} & Y_{22}
\end{array}\right]=\left[\begin{array}{ll}
0 & 0 \\
0 & 0
\end{array}\right],}
\end{aligned}
$$

where $Z$ and $Y$ are the unknown $(2 n+1)$-by- $(2 m+1)$ matrices.

After performing the matrix multiplication, we have

$$
\begin{gathered}
{\left[\begin{array}{cc}
Z_{12} F_{m}+F_{n}^{T} Y_{21} & Z_{11} F_{m}^{T}+F_{n}^{T} Y_{22} \\
Z_{22} F_{m}+F_{n} Y_{11} & Z_{21} F_{m}^{T}+F_{n} Y_{12}
\end{array}\right]=\left[\begin{array}{ll}
0 & 0 \\
0 & 0
\end{array}\right],} \\
{\left[\begin{array}{cc}
Z_{12} G_{m}+G_{n}^{T} Y_{21} & Z_{11} G_{m}^{T}+G_{n}^{T} Y_{22} \\
Z_{22} G_{m}+G_{n} Y_{11} & Z_{21} G_{m}^{T}+G_{n} Y_{12}
\end{array}\right]=\left[\begin{array}{ll}
0 & 0 \\
0 & 0
\end{array}\right] .}
\end{gathered}
$$

It is enough to consider the subsystems that correspond to blocks $(1,1),(2,1)$, and $(2,2)$. Consider first the $(1,1)$-blocks:

$$
\begin{aligned}
& Z_{12} F_{m}+F_{n}^{T} Y_{21}=0 \\
& Z_{12} G_{m}+G_{n}^{T} Y_{21}=0
\end{aligned}
$$

From the first equation of (3.5) we have

$$
Z_{12}=\left[\begin{array}{ccc} 
& -W & \\
0 & \ldots & 0
\end{array}\right], \quad Y_{21}=\left[\begin{array}{cc} 
& 0 \\
W & \vdots \\
& 0
\end{array}\right],
$$

where $W=\left[w_{i j}\right]$ is any $n$-by- $m$ matrix. Substituting $Z_{12}$ and $Y_{21}$ in the second equation of (3.5), we obtain

$$
\left[\begin{array}{ccccc}
0 & w_{11} & \ldots & w_{1, m-1} & w_{1 m} \\
-w_{11} & w_{21}-w_{12} & \ldots & w_{2, m-1}-w_{1 m} & w_{2 m} \\
\ldots \ldots \ldots \ldots \ldots \ldots \ldots \ldots \ldots \ldots \ldots \ldots \ldots \ldots \ldots \ldots \ldots \ldots \\
-w_{n-1,1} & w_{n 1}-w_{n-1,2} & \ldots & w_{n, m-1}-w_{n-1, m} & w_{n m} \\
-w_{n 1} & -w_{n 2} & \ldots & -w_{n m} & 0
\end{array}\right]=0 .
$$

Therefore, we have that $W=0$, and thus, both $Y_{21}$ and $Z_{12}$ are zero blocks.

The subsystem corresponding to the $(2,1)$-blocks is

$$
\begin{aligned}
& Z_{22} F_{m}+F_{n} Y_{11}=0, \\
& Z_{22} G_{m}+G_{n} Y_{11}=0 .
\end{aligned}
$$


By [14, Section 3.4], it has the solution $Z_{22}=-P_{n m}^{\leftrightarrow}$ and $Y_{11}=P_{n+1, m+1}^{\leftrightarrow}$. Recall that the $n \times m$ part, starting form the top-left corner of $Y_{11}$ is equal to $-Z_{22}$ (because of the first equation of (3.6) $)$. Therefore, the parameter entries of both matrices are the same and the dimension of the solution space is equal to $m-n+1$. Note that the subsystem corresponding to the $(1,2)$-blocks is equal to (3.6) up to the transposition and interchanging the roles of $n$ and $m$. Thus, we have that $Y_{22}=\left(-P_{m n}^{\leftrightarrow}\right)^{T}=-P_{n m}^{\uparrow}$ and $Z_{11}=\left(P_{m+1, n+1}^{\leftrightarrow}\right)^{T}=P_{n+1, m+1}^{\uparrow}$ and the dimension of the solution space is equal to $n-m+1$.

Now consider the subsystem of equations corresponding to the $(2,2)$-blocks

$$
\begin{aligned}
& Z_{21} F_{m}^{T}+F_{n} Y_{12}=0, \\
& Z_{21} G_{m}^{T}+G_{n} Y_{12}=0 .
\end{aligned}
$$

From the first equation of (3.7) we obtain that

$$
Y_{12}=\left[\begin{array}{ccc} 
& -W & \\
a_{1} & \cdots & a_{m}
\end{array}\right] \text { and } Z_{21}=\left[\begin{array}{cc} 
& b_{1} \\
W & \vdots \\
& b_{n}
\end{array}\right],
$$

where $W=\left[w_{i j}\right]$ is any $n$-by- $m$ matrix. Substituting $Z_{21}$ and $Y_{12}$ in the second equation of (3.7), we obtain

$$
\left[\begin{array}{ccccc}
w_{21}-w_{12} & w_{22}-w_{13} & \ldots & w_{2, m-1}-w_{1 m} & w_{2 m}-b_{1} \\
w_{31}-w_{22} & w_{32}-w_{23} & \ldots & w_{3, m-1}-w_{2 m} & w_{3 m}-b_{2} \\
\ldots \ldots \ldots \ldots \ldots \ldots \ldots \ldots \ldots \ldots \ldots \ldots \ldots \ldots \ldots \ldots \ldots \ldots \ldots \ldots \ldots \ldots \ldots \\
w_{n 1}-w_{n-1,2} & w_{n 2}-w_{n-1,3} & \ldots & w_{n, m-1}-w_{n-1, m} & w_{n m}-b_{m-1} \\
-a_{1}-w_{n 2} & -a_{2}-w_{n 3} & \ldots & -a_{n-1}-w_{n m} & -a_{n}-b_{m}
\end{array}\right]=0 .
$$

Thus, we have that $Y_{12}=P_{n+1, m}($ an $(n+1) \times m$ dense Hankel matrix $), Z_{21}=-P_{n, m+1}$ (an $n \times(m+1)$ dense Hankel matrix), with common $n \times m$ part (up to the sign, see (3.8) ), and $a_{n}=b_{m}$. Hence, the sets of parameter entries of $Y_{12}$ and $Z_{21}$ are the same, and so they are denoted by the same letter. Calculating the number of independent parameters in the solution we obtain that the dimension of the solution space is equal to $n+m$.

Summing up the answers for all the four equations, we obtain $(Z, Y)$ that is the solution of $\operatorname{syst}\left(L_{n}, L_{m}\right)$ and transposing $Z$ we get

$$
Z^{T}=\left[\begin{array}{cc}
R_{n+1, m+1}^{\downarrow T} & -Q_{m+1, n} \\
0_{m, n+1} & -P_{n m}^{\leftrightarrow}
\end{array}\right] \quad \text { and } \quad Y=\left[\begin{array}{cc}
P_{n+1, m+1}^{\leftrightarrow} & Q_{n+1, m} \\
0_{n, m+1} & -R_{n m}^{\uparrow}
\end{array}\right],
$$

which proves (2.14) due to Lemma 3.1. Calculating the number of independent parameters we obtain

$$
d_{L L}= \begin{cases}2 n+2 & \text { if } n=m \\ 2 \max (n, m)+1 & \text { if } n \neq m\end{cases}
$$


3.5. Solution of $\operatorname{syst}\left(H_{n}(\lambda), K_{m}\right)$. In this section, we compute off-diagonal blocks of the solution of system (1.1) that correspond to the diagonal blocks $H_{n}(\lambda)$ and $K_{m}$. The system $\operatorname{syst}\left(H_{n}(\lambda), K_{m}\right)$ has the form

$$
Z \Lambda_{m}(0)+\Delta_{n} Y=0, \quad Z \Delta_{m}+\Lambda_{n}(\lambda) Y=0
$$

where $Z$ and $Y$ are the unknown $n$-by- $m$ matrices.

From the first equation we have $Y=-\Delta_{n} Z \Lambda_{m}(0)$. We substitute this value of $Y$ in the second equation, multiply the equation by $\Delta_{m}$ from the right side, and use that $\Delta_{m} \Delta_{m}=I_{m}$ and $\Lambda_{m}(\lambda) \Delta_{m}=J_{m}(\lambda)^{T}$ to obtain the following system

$$
Y+\Delta_{n} Z \Lambda_{m}(0)=0, \quad Z-J_{n}(\lambda)^{T} Z J_{m}(0)^{T}=0 .
$$

By [14, Section 3.5] the second equation of the system has only the trivial solution $Z=0$. Thus, $Y=0$ too. Now Lemma 3.1 ensures (2.15). We have no parameters in the solution, so $d_{H K}=0$.

3.6. Solution of $\operatorname{syst}\left(H_{n}(\lambda), L_{m}\right)$. In this section, we find the off-diagonal blocks of the solution of system (1.1) that correspond to the diagonal blocks $H_{n}(\lambda)$ and $L_{m}$. The system $\operatorname{syst}\left(H_{n}(\lambda), L_{m}\right)$ has the form

$$
\begin{array}{r}
Z\left[\begin{array}{cc}
0 & F_{m}^{T} \\
F_{m} & 0
\end{array}\right]+\Delta_{n} Y=0 \\
Z\left[\begin{array}{cc}
0 & G_{m}^{T} \\
G_{m} & 0
\end{array}\right]+\Lambda_{n}(\lambda) Y=0,
\end{array}
$$

where $Z$ and $Y$ are the unknown $n$-by- $(2 m+1)$ matrices.

From the first equation we have

$$
\left[\begin{array}{ll}
Y_{1} & Y_{2}
\end{array}\right]=\left[\begin{array}{ll}
-\Delta_{n} Z_{2} F_{m} & -\Delta_{n} Z_{1} F_{m}^{T}
\end{array}\right]
$$

Substituting it in the second equation, we obtain

$$
\left[Z_{2} G_{m}-J_{n}(\lambda)^{T} Z_{2} F_{m} \quad Z_{1} G_{m}^{T}-J_{n}(\lambda)^{T} Z_{1} F_{m}^{T}\right]=0 .
$$

The solutions of these equations are given in [14, Section 3.6]. Altogether, using (3.9) we have the solution $(Z, Y)$ of $\operatorname{syst}\left(H_{n}(\lambda), L_{m}\right)$ and therefore

$$
Z^{T}=\left[\begin{array}{c}
-P_{m+1, n}^{\ulcorner}(\lambda) \\
0_{m n}
\end{array}\right] \quad \text { and } \quad Y=\left[\begin{array}{ll}
0_{n, m+1} & \Delta_{n} P_{m+1, n}^{\ulcorner T}(\lambda) F_{m}^{T}
\end{array}\right],
$$

which together with Lemma 3.1 ensures (2.16). The number of independent parameters in the solution is $d_{H L}=2 n$. 
3.7. Solution of $\operatorname{syst}\left(K_{n}, L_{m}\right)$. Finally, we compute off-diagonal blocks of the solution of system (1.1) that correspond to the diagonal blocks $K_{n}$ and $L_{m}$. The $\operatorname{system} \operatorname{syst}\left(K_{n}, L_{m}\right)$ has the form

$$
\begin{gathered}
Z\left[\begin{array}{cc}
0 & F_{m}^{T} \\
F_{m} & 0
\end{array}\right]+\Lambda_{n}(0) Y=0 \\
Z\left[\begin{array}{cc}
0 & G_{m}^{T} \\
G_{m} & 0
\end{array}\right]+\Delta_{n} Y=0
\end{gathered}
$$

where $Z$ and $Y$ are the unknown $n$-by- $(2 m+1)$ matrices.

From the second equation we obtain

$$
\left[\begin{array}{ll}
Y_{1} & Y_{2}
\end{array}\right]=\left[\begin{array}{ll}
-\Delta_{n} Z_{2} G_{m} & -\Delta_{n} Z_{1} G_{m}^{T}
\end{array}\right]
$$

By substituting it in the first equation, we have

$$
\left[Z_{2} F_{m}-J_{n}(0)^{T} Z_{2} G_{m} \quad Z_{1} F_{m}^{T}-J_{n}(0)^{T} Z_{1} G_{m}^{T}\right]=0 .
$$

The solutions of these equations are given in [14, Section 3.7]. Using (3.10) and transposing $Z$ we get

$$
Z^{T}=\left[\begin{array}{c}
-P_{n, m+1}^{\Downarrow T} \\
0_{m n}
\end{array}\right] \text { and } Y=\left[\begin{array}{ll}
0_{n, m+1} & \Delta_{n} P_{n, m+1}^{\Downarrow} G_{m}^{T}
\end{array}\right] .
$$

Thus, using Lemma 3.1, we prove (2.17). Calculating the number of independent parameters in the solution we obtain that $d_{K L}=2 n$.

4. Two examples. We illustrate our results by considering two different pairs of matrix equations (1.1) with $(A, B)$ in canonical form.

EXAMPle 4.1. Consider the system (1.1) given by the symmetric $13 \times 13$ matrix pair $(A, B)=K_{3} \oplus L_{1} \oplus L_{3}$. By Theorem 2.1 its solution is the matrix

$$
X=\left[\begin{array}{ccc|cc:c|cccc:ccc}
0 & 0 & 0 & 0 & 0 & x_{3} & 0 & 0 & 0 & 0 & x_{5} & x_{6} & x_{7} \\
0 & 0 & 0 & 0 & 0 & x_{2} & 0 & 0 & 0 & 0 & 0 & x_{5} & x_{6} \\
0 & 0 & 0 & 0 & 0 & x_{1} & 0 & 0 & 0 & 0 & 0 & 0 & x_{5} \\
\hline 0 & -x_{1} & -x_{2} & x_{4} & 0 & 0 & x_{13} & x_{14} & x_{15} & 0 & x_{8} & x_{9} & x_{10} \\
-x_{1} & -x_{2} & -x_{3} & 0 & x_{4} & 0 & 0 & x_{13} & x_{14} & x_{15} & x_{9} & x_{10} & x_{11} \\
\hdashline 0 & 0 & 0 & 0 & 0 & -x_{4} & 0 & 0 & 0 & 0 & 0 & 0 & 0 \\
\hline 0 & 0 & 0 & 0 & 0 & -x_{8} & x_{12} & 0 & 0 & 0 & 0 & 0 & 0 \\
0 & 0 & -x_{5} & 0 & 0 & -x_{9} & 0 & x_{12} & 0 & 0 & 0 & 0 & 0 \\
0 & -x_{5} & -x_{6} & 0 & 0 & -x_{10} & 0 & 0 & x_{12} & 0 & 0 & 0 & 0 \\
-x_{5} & -x_{6} & -x_{7} & 0 & 0 & -x_{11} & 0 & 0 & 0 & x_{12} & 0 & 0 & 0 \\
\hdashline 0 & 0 & 0 & 0 & 0 & -x_{13} & 0 & 0 & 0 & 0 & -x_{12} & 0 & 0 \\
0 & 0 & 0 & 0 & 0 & -x_{14} & 0 & 0 & 0 & 0 & 0 & -x_{12} & 0 \\
0 & 0 & 0 & 0 & 0 & -x_{15} & 0 & 0 & 0 & 0 & 0 & 0 & -x_{12}
\end{array}\right],
$$


in which $x_{1}, \ldots, x_{15} \in \mathbb{C}$.

The dimension of the solution space is $d_{(A, B)}=15$ (the number of independent parameters in $X)$, and therefore, the codimension of the congruence orbit of $(A, B)$ is equal to $d_{(A, B)}+\hat{n}=28$.

EXAmple 4.2. Consider the system (1.1) given by the symmetric $7 \times 7$ matrix pair $(A, B)=H_{2}(\lambda) \oplus L_{2}$. By Theorem 2.1]its solution is the matrix

$$
X=\left[\begin{array}{cc|ccc:cc}
0 & 0 & 0 & 0 & 0 & x_{2} & \lambda x_{2}+x_{1} \\
0 & 0 & 0 & 0 & 0 & x_{1} & \lambda x_{1} \\
\hline-x_{1} & -x_{2} & x_{3} & 0 & 0 & 0 & 0 \\
-\lambda x_{1} & -\lambda x_{2}-x_{1} & 0 & x_{3} & 0 & 0 & 0 \\
-\lambda^{2} x_{1} & -\lambda^{2} x_{2}-2 \lambda x_{1} & 0 & 0 & x_{3} & 0 & 0 \\
\hdashline 0 & 0 & 0 & 0 & 0 & -x_{3} & 0 \\
0 & 0 & 0 & 0 & 0 & 0 & -x_{3}
\end{array}\right],
$$

in which $x_{1}, x_{2}, x_{3} \in \mathbb{C}$.

The dimension of the solution space is $d_{(A, B)}=3$ (the number of independent parameters in $X)$, and therefore, the codimension of the congruence orbit of $(A, B)$ is equal to $d_{(A, B)}+\hat{n}=10$.

\section{REFERENCES}

[1] M.A. Beitia and J.M. Gracia. Sylvester matrix equation for matrix pencils. Linear Algebra Appl., 232:155-197, 1996.

[2] W.M. Boothby. An Introduction to Differentiable Manifolds and Riemannian Geometry. Academic Press, New York, 1975.

[3] T. Brüll and V. Mehrmann. STCSSP: A FORTRAN 77 Routine to Compute a Structured Staircase Form for a (Skew-)Symmetric/(Skew-)Symmetric Matrix Pencil. Preprint 312007, Institut für Mathematik, TU Berlin, 2007.

[4] A.Z.-Y. Chan, L.A. Garcia German, S.R. Garcia, and A.L. Shoemaker. On the matrix equation $X A+A X^{T}=0$, II: Type 0-I interactions. Linear Algebra Appl., 439:3934-3944, 2013.

[5] F. De Terán. The solution of the equation $A X+B X^{\star}=0$. Linear Multilinear Algebra, 61:16051628, 2013.

[6] F. De Terán and F.M. Dopico. The equation $X A+A X^{*}=0$ and the dimension of *congruence orbits. Electron. J. Linear Algebra, 22:448-465, 2011.

[7] F. De Terán and F.M. Dopico. The solution of the equation $X A+A X^{T}=0$ and its application to the theory of orbits. Linear Algebra Appl., 434:44-67, 2011.

[8] F. De Terán, F.M. Dopico, N. Guillery, D. Montealegre, and N. Reyes. The solution of the equation $A X+X^{\star} B=0$. Linear Algebra Appl., 438:2817-2860, 2013.

[9] A. Dmytryshyn. Miniversal Deformations of Pairs of Skew-Symmetric Forms. Master Thesis, Kiev National University, Kiev, arXiv:1104.2492, 2010.

[10] A. Dmytryshyn. Miniversal deformations of pairs of symmetric forms. Preprint, arXiv:1104.2530, 2011.

[11] A.R. Dmytryshyn, V. Futorny, and V.V. Sergeichuk. Miniversal deformations of matrices of bilinear forms. Linear Algebra Appl., 436:2670-2700, 2012. 
[12] A. Dmytryshyn, V. Futorny, and V.V. Sergeichuk. Miniversal deformations of matrices under *congruence and reducing transformations Linear Algebra Appl., to appear, 2014, DOI: 10.1016/j.laa.2014.01.016.

[13] A. Dmytryshyn, S. Johansson, and B. Kågström. Codimension Computations of Congruence Orbits of Matrices, Skew-Symmetric and Symmetric Matrix Pencils Using Matlab. Technical report UMINF 13.18, Department of Computing Science, Umeå University, Sweden, 2013.

[14] A. Dmytryshyn, B. Kågström, and V.V. Sergeichuk. Skew-symmetric matrix pencils: Codimension counts and the solution of a pair of matrix equations. Linear Algebra Appl., 438:3375-3396, 2013.

[15] N.A. Dumont. On the solution of generalized non-linear complex-symmetric eigenvalue problems. Internat. J. Numer. Methods Engrg., 71:1534-1568, 2007.

[16] A. Edelman, E. Elmroth, and B. Kågström. A geometric approach to perturbation theory of matrices and matrix pencils. Part I: Versal deformations. SIAM J. Matrix Anal. Appl., 18:653-692, 1997.

[17] A. Edelman, E. Elmroth, and B. Kågström. A geometric approach to perturbation theory of matrices and matrix pencils. Part II: A stratification-enhanced staircase algorithm. SIAM J. Matrix Anal. Appl., 20:667-669, 1999.

[18] F.R. Gantmacher. The Theory of Matrices. Chelsea Publishing Co., New York, 1959.

[19] S.R. Garcia and A.L. Shoemaker. On the matrix equation $X A+A X^{T}=0$. Linear Algebra Appl., 438:2740-2746, 2013.

[20] M.I. Garcia-Planas and V.V. Sergeichuk. Simplest miniversal deformations of matrices, matrix pencils, and contragredient matrix pencils. Linear Algebra Appl., 302/303:45-61, 1999.

[21] N.J. Higham, D.S. Mackey, N. Mackey, and F. Tisseur. Symmetric linearizations for matrix polynomials. SIAM J. Matrix Anal. Appl., 29:143-159, 2006.

[22] P. Johansson. Matrix Canonical Structure Toolbox. Technical report UMINF 06.15, Department of Computing Science, Umeå University, Sweden, 2006.

[23] B. Kågström, S. Johansson, and P. Johansson. StratiGraph tool: Matrix stratifications in control applications. In: L. Biegler, S. L. Campbell, and V. Mehrmann (editors), Control and Optimization with Differential-Algebraic Constraints. SIAM Publications, Philadelphia, 79-103, 2012.

[24] B. Kågström, L. Karlsson, and D. Kressner. Computing codimensions and generic canonical forms for generalized matrix products. Electron. J. Linear Algebra, 22:277-309, 2011.

[25] B.N. Parlett. Symmetric matrix pencils. J. Comput. Appl. Math., 38:373-385, 1991.

[26] A. Tannenbaum. Invariance and System Theory: Algebraic and Geometric Aspects. Lecture Notes in Mathematics, Vol. 845, Springer-Verlag, Berlin - New York, 1981.

[27] R.C. Thompson. Pencils of complex and real symmetric and skew matrices. Linear Algebra Appl., 147:323-371, 1991.

[28] F. Tisseur and K. Meerbergen. The quadratic eigenvalue problem. SIAM Rev., 43:235-286, 2001. 